\section{Uso de órtese para abdução do polegar no desempenho funcional de criança portadora de paralisia cerebral: estudo de caso único}

\author{
Use of abduction thumb orthosis in \\ functional performance of a child with \\ cerebral palsy: a single-subject study
}

Adriana M. Valladão N. Rodrigues ${ }^{1}$

Marisa C. Mancini ${ }^{2}$

Daniela V. Vaz ${ }^{3}$

Lílian de Castro Silva ${ }^{4}$

1,2,4 Departamento de Terapia Ocupacional. Universidade Federal de Minas Gerais. Av. Antônio Carlos, 6627. Belo Horizonte, MG, Brasil. CEP: 31.270-901. E-mail: avaladao@ufmg.br

3 Departamento de Fisioterapia. Universidade Federal de Minas Gerais. Belo Horizonte, MG, Brasil.

\begin{abstract}
Objective: to investigate the impact of a thumb abduction orthosis on functional skills of a child with hemiparetic cerebral palsy and mild spasticity.

Methods: an $A B$ single-subject design was used in this study. Baseline A consisted of data collected during a period without intervention (control conditions). The intervention phase B included the use of a thumb abduction orthosis in association with traditional occupational therapy. The baseline lasted six weeks and the intervention period eight weeks. Assessments were performed once a week throughout the study and included hand function tests and range of motion measures for active wrist extension and flexion and thumb abduction and opponency. The Celeration Line and Two-Standard Deviation Band methods, as well as Visual Analysis, were used for data analysis.

Results: the child demonstrated significant improvements in the range of active motions. A trend towards a reduction in the time to perform manual tasks was observed, but this was only statistically significant in the case of the task of stacking blocks.

Conclusion: the results suggest that thumb abduction orthosis may be a useful adjunct treatment to improve the range of active hand motions in children with hemiparetic cerebral palsy.
\end{abstract}

Key words Orthosis, Thumb, Cerebral palsy

\section{Resumo}

Objetivos: documentar o impacto do uso da órtese de abdução do polegar no desempenho funcional de uma criança hemiparética com leve espasticidade.

Métodos: foi utilizado um desenho experimental de caso-único do tipo AB. A fase A consistiu na coleta de dados durante um período sem intervenção (condição controle). A fase B incluiu o uso de órtese de abdução do polegar como procedimento terapêutico associado ao atendimento de terapia ocupacional tradicional. A fase A teve a duração de seis semanas e a fase subseqüente de oito semanas. A criança foi avaliada semanalmente quanto à amplitude ativa de movimento de extensão e flexão de punho, abdução e oponência do polegar e quanto à função manual. A análise dos dados foi realizada através dos métodos estatísticos Celeration Line e Banda de Dois Desvios-Padrão, assim como da Análise Visual.

Resultados: a criança apresentou melhora significativa na amplitude de movimento do punho e polegar (ADM ativa). Com relação à função manual observouse redução do tempo para realização das tarefas, porém não significativa, exceto para a tarefa de empilhar blocos.

Conclusões: os resultados sugerem que o uso da órtese de abdução do polegar pode ser útil no tratamento de crianças com paralisia cerebral espástica para a melhoria da ADM ativa da mão, podendo ser utilizada como adjuvante a outras terapêticas.

Palavras-chave Órtese, Polegar, Paralisia cerebral 


\section{Introdução}

A mão é uma ferramenta que tem como propósito primário a exploração e manipulação de objetos. ${ }^{1} \mathrm{O}$ desenvolvimento das habilidades manuais é essencial para o desempenho da criança nas tarefas de brincar e nas atividades de vida diária. ${ }^{2}$

O tamanho, a força, o movimento lateral livre e a perfeita mobilidade do polegar influenciam diretamente o poder da mão humana. ${ }^{3}$ A posição do polegar em abdução e oposição é constante na maioria dos tipos de preensão e se mantém inalterada a despeito do tamanho ou forma do objeto ${ }^{4}$ oferecendo ao homem uma destreza manual incomparável. ${ }^{5}$ Segundo Fess e Philips, ${ }^{5}$ quando há a perda do polegar o déficit funcional da mão apresenta-se em torno de $40 \%$, porém a real perda observada pode ser substancialmente maior. Portanto, a importância do polegar na maioria dos aspectos da função da mão não pode ser desconsiderada.

Muitas crianças com paralisia cerebral apresentam problemas de controle motor, resultante de lesões primárias ou secundárias envolvendo o córtex sensório-motor e o trato cortical, o que pode gerar um sério impacto na habilidade funcional da extremidade superior. ${ }^{6,7}$ Em geral, essas crianças apresentam tônus muscular, padrão de movimento e posturas anormais das extremidades. ${ }^{8}$ Os padrões de movimentos típicos da extremidade superior da criança hemiparética podem incluir rotação interna do ombro, flexão do cotovelo com antebraço em pronação, desvio ulnar, flexão do punho e adução e flexão do polegar. ${ }^{9}$ Esses padrões influenciam diretamente na precisão da preensão e independência dos movimentos dos dedos. 7

A adução e flexão excessivas do polegar, que caracterizam o chamado polegar incluso, limitam a função da mão, especialmente nas preensões palmar e fina, podendo também resultar em incapacidades, ou seja, limitações no desempenho de atividades e tarefas do cotidiano da criança. Essas tarefas incluem atividades escolares, como manipulação de objetos e atividades de auto-cuidado, tais como alimentar-se, vestir-se e realizar tarefas de higiene pessoal. ${ }^{10}$

Nestes casos, o tratamento intensivo com enfoque na função manual deve ser planejado precocemente, tendo em vista que a experiência da exploração de novas sensações, envolvendo o manipular, é de extrema importância para o desenvolvimento motor, social e cognitivo da criança. ${ }^{11}$ Como coadjuvante no tratamento, o uso de diferentes tipos de órteses é indicado para minimizar as limitações impostas pela rigidez encontrada no padrão de adução do polegar. ${ }^{11}$ Este tipo de órtese abre o primeiro espaço interdigital e posiciona o polegar em abdução e extensão com oponência e mantém o arco palmar adequadamente, ${ }^{12}$ a fim de permitir a melhora da qualidade de movimento e prevenção ou correção de deformidades. ${ }^{5,11,13}$ A órtese para abdução do polegar é indicada para crianças com paralisia cerebral hemiparética com espasticidade leve a moderada na mão e permite a utilização do polegar na preensão de objetos, contribuindo para um aumento significativo dos aspectos fundamentais da função manual. ${ }^{10}$

Embora uma significativa porção da função manual seja atribuída ao polegar, é curioso que tão pouca bibliografia referente à biomecânica do uso de órteses para o polegar seja encontrada, ${ }^{3}$ principalmente com crianças com paralisia cerebral, já que o uso desse dispositivo pode maximizar a função manual. O objetivo deste estudo foi documentar os efeitos da órtese de abdução do polegar na amplitude de movimento ativa de articulações da mão e na função manual de uma criança com idade de três anos com hemiparesia espástica leve.

\section{Métodos}

Neste estudo foi utilizado um desenho experimental de caso único do tipo $\mathrm{AB}$, que permite a comparação sistemática entre as fases de baseline $(A)$ e intervenção $(B)$. A fase de baseline consistiu na coleta de dados durante um período sem intervenção (condição controle). A fase B incluiu o uso de órtese de abdução do polegar como procedimento terapêutico, associado ao atendimento de terapia ocupacional tradicional. Os desfechos avaliados foram a amplitude de movimento ativa de flexão e extensão de punho, abdução e oponência do polegar e o desempenho funcional durante o teste de função manual de Jebsen-Taylor.

O estudo contou com a participação de uma criança de três anos de idade, do sexo feminino com paralisia cerebral. Os critérios de inclusão utilizados para seleção do participante foram: hemiparesia; grau de espasticidade $(+1)$ de acordo com a Escala de Ashworth; ${ }^{14}$ adução do polegar que limitava a realização de atividades de preensão, pinça fina, a coordenação do pegar, liberação de objetos e a realização de atividades bimanuais; utilização da mão acometida principalmente como membro auxiliar durante a realização das atividades; certo controle voluntário dos movimentos de extensão e flexão do punho. A criança deveria ainda estar em atendimento terapêutico ocupacional. 
Para participação no estudo, a criança não poderia apresentar quaisquer outras patologias associadas; não poderia se submeter à outros tratamentos além da terapia ocupacional e não deveria apresentar déficit percepto-cognitivo que a impedisse de colaborar nos procedimentos do estudo.

A participante estava em atendimento e acompanhamento no Setor de Terapia Ocupacional Pediatria do anexo Ambulatório Bias Fortes do Complexo Hospital das Clínicas da Universidade Federal de Minas Gerais e foi indicada para participação no estudo pelos profissionais do serviço, e submetida à avaliação para verificação de sua adequação aos critérios de inclusão previstos.

Três métodos de avaliação foram utilizados: a Escala de Ashworth, ${ }^{14}$ o teste de amplitude de movimento ativa (goniometria) e o Teste de Função Manual de Jebsen-Taylor.

A Escala de Ashworth ${ }^{14}$ é indicada para avaliar o grau de tônus muscular do indivíduo. O tônus pode ser graduado em cinco níveis, de 0 (zero) quando não se observa qualquer aumento de tônus, até 4 (quatro) quando o membro apresenta-se rígido na flexão ou extensão. Nesse estudo, esse instrumento foi utilizado apenas na avaliação inicial do participante para inserção ou não do mesmo na pesquisa.

O Teste de Amplitude de Movimento (ADM) articular consiste na medida do arco de movimento através do qual passa uma articulação ${ }^{15}$ e foi realizada, no estudo, utilizando-se um goniômetro universal para pequenas e médias articulações para medida da ADM ativa de flexão e extensão de punho e abdução do polegar. Optou-se por avaliar apenas a ADM ativa de movimento já que o maior interesse era avaliar o desempenho funcional da mão. Para a medida da oponência do polegar utilizou-se a medida da distância entre as polpas digitais do polegar e $\mathrm{V}$ dedo, através do uso de uma régua com medida em centímetros. ${ }^{16}$

O teste da função manual de Jebsen-Taylor é um teste padronizado, desenvolvido para avaliar o uso funcional da mão. ${ }^{17,18}$ Trata-se de um instrumento de fácil administração que tem como objetivos proporcionar uma medida objetiva de tarefas padronizadas, avaliar alguns aspectos da função da mão comumente usados em atividades do cotidiano e documentar continuamente a habilidade em cada categoria da função da mão testada. ${ }^{19}$ Esse teste é composto por sete sub-testes: escrita, virar cartas, pegar pequenos objetos, simulação de alimentação, empilhar blocos, pegar objetos leves e pegar objetos pesados. Os escores dos sub-testes consistiram nas mensurações dos tempos, através de cronômetro Digital Readout Stopwatch - necessários para completar cada uma das tarefas. As atividades deviam ser realizadas depois que as instruções fossem dadas e após certificar-se que as mesmas foram compreendidas pela criança. Os sub-testes foram sempre apresentados na mesma seqüência e realizados inicialmente com a mão não dominante, conforme preconizado por Jebsen et al. ${ }^{19}$ Ressalta-se que nesse estudo, devido à idade da criança, os subtestes de escrita e simulação de alimentação não foram utilizados. ${ }^{20}$

Os dados foram coletados por 14 semanas, durante as quais a criança manteve suas atividades regulares e esteve em tratamento terapêutico ocupacional. Nesse período a criança foi submetida à avaliação para caracterização do desempenho funcional e da amplitude de movimento na fase de baseline $(A)$ nas primeiras seis semanas do estudo, $\mathrm{e}$ nas oito semanas da fase de intervenção $(B)$ em que fez uso da órtese. Ao longo do período do estudo a participante foi avaliada semanalmente, no mesmo dia do atendimento terapêutico ocupacional agendado pelo serviço (antes do atendimento).

Para a realização do teste Jebsen-Taylor a criança foi posicionada sentada, de frente para uma mesa e instruída a realizar o mais rápido possível os cinco sub-testes definidos. Cada tarefa (sub-teste) foi iniciada após o comando verbal da examinadora e o tempo necessário para completá-la (o final da tarefa foi definido como o momento de liberação do último objeto), foi registrado com um cronômetro (Digital Readout Stopwatch). Em caso de erro (deixar cair o objeto, distração, etc.), correções foram realizadas e a atividade foi repetida imediatamente. ${ }^{21}$

Para a avaliação da ADM ativa de extensão e flexão do punho, o goniômetro foi posicionado com o braço fixo paralelo ao antebraço da criança, o braço móvel paralelo ao segundo metacarpiano e o eixo sobre a articulação do punho. A criança foi posicionada sentada ao lado da mesa, com o antebraço sobre a mesa, estabilizado em posição neutra pela examinadora e, em seguida, foi instruída e encorajada a mover seu punho até alcançar o máximo de extensão. $\mathrm{O}$ mesmo procedimento foi mantido para a medida da máxima flexão ativa do punho. Para a avaliação da ADM ativa de abdução do polegar, o goniômetro foi posicionado com o braço fixo paralelo ao segundo metacarpiano, braço móvel paralelo primeiro metacarpiano e o eixo na articulação carpometacarpo do polegar. $\mathrm{O}$ antebraço e punho foram mantidos em posição neutra e o polegar foi posicionado em alinhamento completo com o segundo metacarpiano, como posição inicial para a mensuração da ADM ativa máxima de abdução do polegar. Para a documentação da oponência utilizou-se a 
medida da distância (centímetros) entre as polpas digitais do polegar e $\mathrm{V}$ dedo.

$\mathrm{Na}$ fase de intervenção, o tratamento terapêutico ocupacional foi mantido acrescido do uso da órtese de abdução do polegar. A órtese para abdução do polegar (Figura 1) foi confeccionada de material termo-moldável (Ezeform $\left.{ }^{\circledR}\right)$ e sob medida para a criança. Essa órtese abre o primeiro espaço interdigital e posiciona o polegar em abdução com oponência. Esse dispositivo tem como objetivo facilitar a preensão e aumentar a amplitude de movimento. Cobre relativamente pequena área da mão, permite boa ventilação da região e deixa a palma livre para a recepção de input sensorial e para a realização de atividades funcionais. ${ }^{12}$ Todas as orientações quanto ao uso, conservação e manutenção da órtese foram repassadas aos responsáveis pela criança. A criança foi orientada a fazer uso diário e constante da órtese, retirando-a apenas para higiene e durante o sono. Ajustes necessários foram realizados na órtese com o objetivo de evitar pontos de preensão e manutenção da postura adequada para função.

Figura 1

Órtese de abdução do polegar.

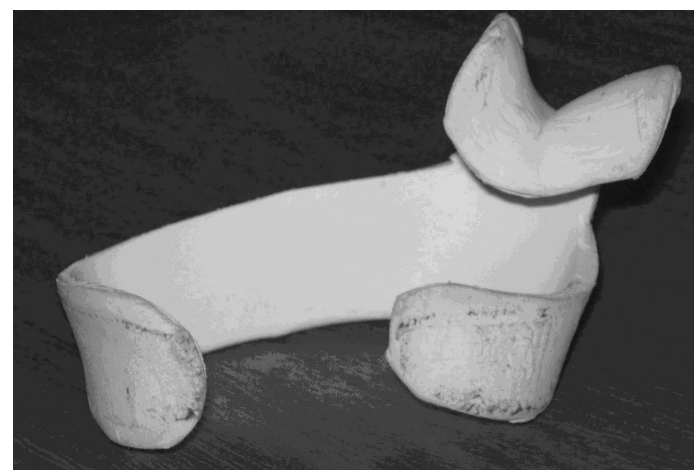

Ressalta-se que a medida da amplitude de movimento foi o primeiro teste aplicado seguido do teste de função manual de Jebsen-Taylor. Essa sequiência foi mantida durante todo o período do estudo.

Antes do início do estudo foi realizado treinamento para administração das medidas de goniometria e do teste de função manual de Jebsen-Taylor e testada a confiabilidade teste-reteste de um único examinador. Os índices de consistência obtidos foram superiores a 0,80 indicando estabilidade dos escores nos dois momentos (intervalo de uma semana). A aplicação dos testes nesse estudo foi realizada somente por esse examinador.
Em estudo experimental de caso único, a análise criteriosa das medidas intra e inter fases é necessária para determinar se houve mudanças de comportamento na fase de intervenção e se estas estão associadas ao tratamento proposto. ${ }^{22}$ No presente estudo, os métodos estatísticos utilizados para analisar os dados referentes aos desfechos investigados foram Celeration Line e Banda de Dois Desvios-Padrão.

A Celeration Line foi utilizada para demonstrar, por meio de uma linha de tendência, a direção da mudança (aceleração, desaceleração ou estabilização) dos dados de uma fase em relação à fase subseqüente. O efeito de medida foi avaliado comparando, entre as duas fases consecutivas, a proporção de dados acima e abaixo da linha de tendência. A significância estatística foi determinada através do teste binomial, considerando o nível de significância de $\alpha=0,05 .^{22}$

O método da Banda de Dois Desvios-Padrão envolveu o cálculo da média e desvios-padrão dos dados da fase de baseline (A). As bandas são desenhadas com mais ou menos dois desvios-padrão em relação à média obtida na fase de baseline, sendo estendidas à fase subseqüente. ${ }^{22}$ A significância estatística é baseada nas premissas de que os dados são independentes e normalmente distribuídos. Considera-se que as mudanças são estatisticamente significativas quando pelo menos dois pontos consecutivos na fase de intervenção encontram-se fora da banda de dois desvios-padrão, uma vez que a probabilidade de que tal evento ocorra é menor do que 0,05 .

$\mathrm{Na}$ presença de discordância entre os dois métodos estatísticos, a análise visual foi utilizada em adição aos métodos estatísticos para decisão de resultado. Na análise visual os dados são descritos de acordo com os níveis e direção das mudanças, bem como o ângulo da inclinação da linha de tendência. Mudanças no nível referem-se ao valor da variável dependente ou magnitude do desempenho no ponto de intervenção. O nível é avaliado pela comparação do último valor obtido do comportamento alvo na primeira fase e o primeiro valor obtido na fase subseqüente. A mudança de nível também pode ser descrita pela comparação do valor médio do comportamento alvo obtido em cada fase.

Neste estudo, esse último método foi utilizado como recurso. Com relação à tendência, ela se refere à mudança de direção em cada fase. Pode ser descrita como aceleração, desaceleração ou estabilização. A inclinação da tendência refere-se ao ângulo ou percentual de mudança entre os dados. A inclinação pode ser determinada por uma linha de tendência linear. Quando o ângulo de inclinação é 
maior na fase de intervenção considera-se que o percentual de mudança do comportamento alvo aumenta quando o tratamento é iniciado. ${ }^{22}$

Registra-se que os procedimentos do presente estudo foram aprovados pelo Comitê de Ética em Pesquisa da Universidade Federal de Minas Gerais (Parecer $\mathrm{n}^{\circ}$ Etic 186/04) e o termo de consentimento livre e esclarecido foi assinado pelos responsáveis anteriormente à inclusão da criança no estudo.

\section{Resultados}

Os resultados dos métodos Celeration Line e Banda de Dois Desvios-Padrão da goniometria do punho, abdução e oponência do polegar são apresentados nas Figuras 2 - 5 e Tabela 1. Uma vez que os valores de abdução do polegar durante a fase de baseline foram todos iguais a zero, para a análise dessa variável foi utilizado somente o método Celeration Line.

Nota-se um aumento das amplitudes de movimento ativo de extensão e flexão do punho, aumento na amplitude de movimento ativo de abdução do polegar e redução total das distâncias entre as polpas digitais possibilitando a oponência total do polegar. A análise dos dados revela que o uso da órtese de polegar (fase de intervenção) teve um efeito estatisticamente significativo nas amplitudes de movimento ativo avaliadas, tanto de acordo com a Celeration Line quanto com o método da Banda de Dois Desvios-Padrão, refletindo grande mudança entre as fases.

Em relação à extensão do punho, os dois métodos estatísticos evidenciam resultados significativos, porém com direções opostas. Enquanto a Banda de Dois Desvios-Padrão identificou melhora significativa na ADM, a Celeration Line evidenciou declínio significativo. No entanto, a análise visual (Figura 2) aponta para uma mudança de nível entre a fase de baseline (média em graus: 34 ) e a fase de intervenção (média em graus: 45,62); a tendência de aceleração é mantida nas duas fases, porém a inclinação da linha de tendência é ligeiramente maior na fase $B$, corroborando os resultados da Banda de Dois Desvios-Padrão, que indica melhora significativa na

Figura 2

Resultados dos métodos estatísticos Celeration Line e Banda de Dois Desvios-Padrão referente à amplitude de movimento ativa de extensão de punho.

\section{Extensão Punho}
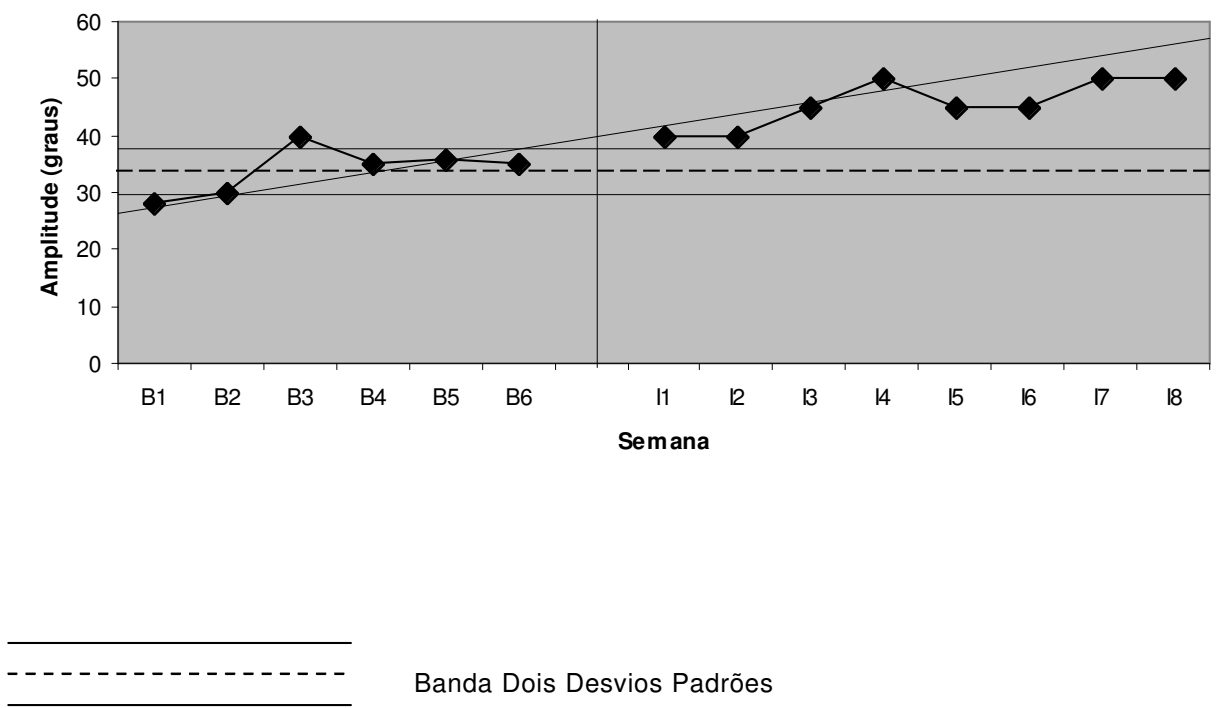

Celeration Line 


\section{Figura 3}

Resultados dos métodos estatísticos Celeration Line e Banda de Dois Desvios-Padrão referente à amplitude de movimento ativa de flexão de punho.

\section{Fexão Punho}

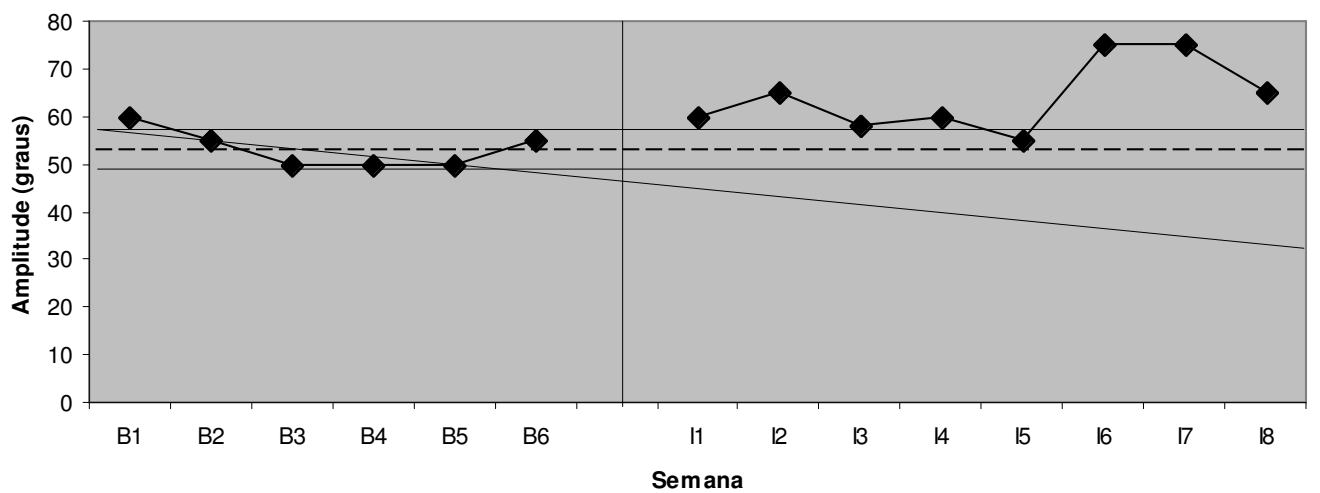

\section{Figura 4}

Resultados dos métodos estatísticos Celeration Line e Banda de Dois Desvios-Padrão referente à amplitude de movimento ativa de abdução do polegar.

\section{Abdução Polegar}
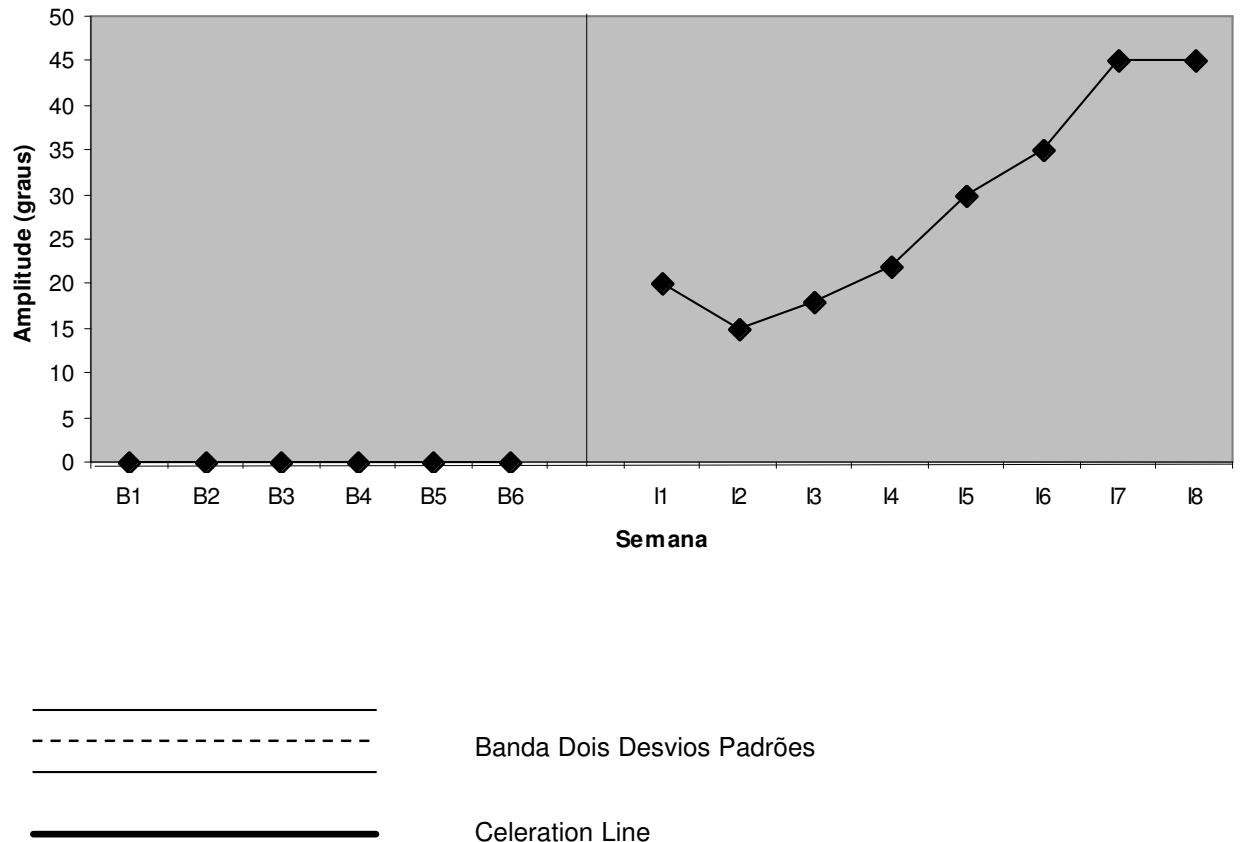

Banda Dois Desvios Padrões

Celeration Line 
Resultados dos métodos estatísticos Celeration Line e Banda de Dois Desvios-Padrão referente à amplitude de movimento ativa de oponência do polegar.

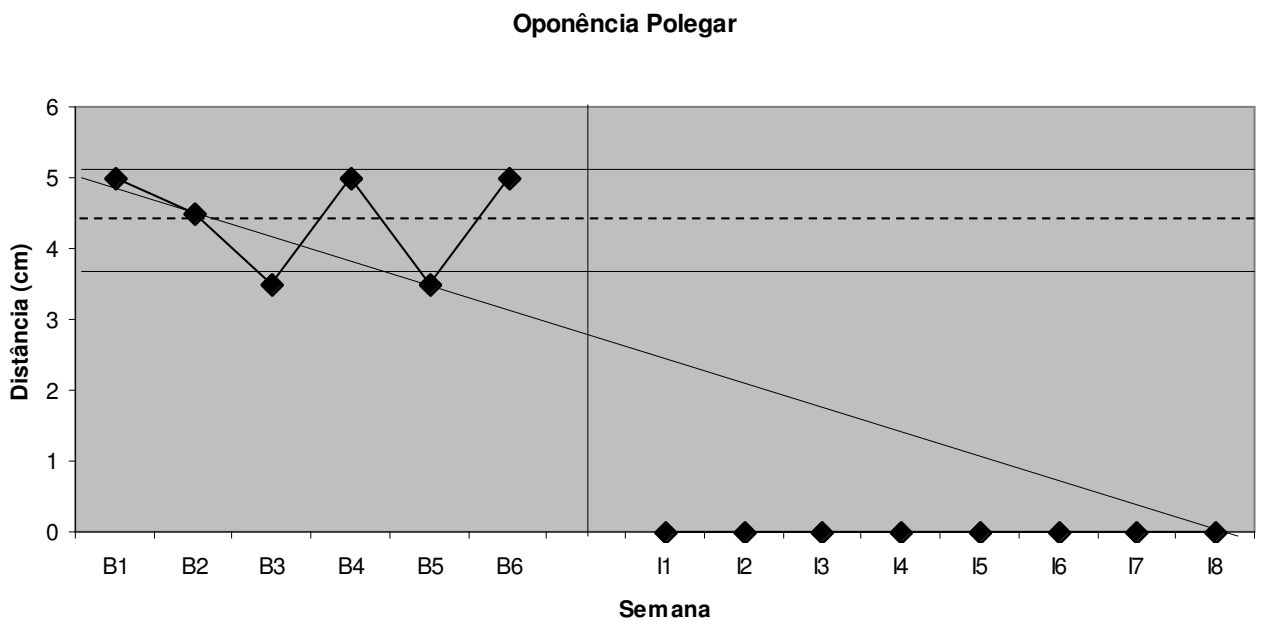

Tabela 1

Análise dos métodos estatísticos Celeration Line e Banda de Dois Desvios-Padrão das variáveis dependentes amplitude de movimento ativa de punho e polegar da criança.

\begin{tabular}{lccc}
\hline Variáveis dependentes & Celeration Line* & Banda de Dois Desvios-Padrão & Análise visual \\
\hline Extensão de e punho & $\mathrm{S} \downarrow(0.035)$ & $\mathrm{S}$ & $\mathrm{S}$ \\
Flexão de punho & $\mathrm{S}(0.004)$ & $\mathrm{S}$ & - \\
Abdução polegar & $\mathrm{S}(0.004)$ & Não se aplica & - \\
Oponência polegar & $\mathrm{S}(0.004)$ & $\mathrm{S}$ & -
\end{tabular}

${ }^{*} p<0,05 ; \mathrm{S}=$ resultado estatisticamente significativo; $\mathrm{S} \downarrow=$ resultado estatisticamente significativo no sentido oposto ao esperado.

ADM ativa de extensão de punho.

As informações contidas na Tabela 1 demonstram que houve a concordância de $66 \%$ entre os métodos estatísticos em todas as variáveis dependentes avaliadas. A concordância entre os dois testes estatísticos foi determinada pela divisão do número de resultados em que os dois testes produziram resultados similares pelo número total de resultados avaliados pelos dois testes, multiplicados por $100 .^{23}$

Os resultados dos métodos Celeration Line e Banda de Dois Desvios-Padrão relacionados ao teste de função manual de Jebsen-Taylor são apresentados nas Figuras 6 - 10 e Tabela 2.

A análise dos dados revelou que na fase de intervenção $(B)$, o desempenho funcional da criança apre- sentou melhoras significativas na tarefa de empilhar blocos, segundo os resultados da Celeration Line. De acordo com a Banda de Dois Desvios-Padrão, a criança obteve melhora significativa no desempenho das tarefas de virar cartas e pegar pequenos objetos. Para a tarefa de pegar objetos pesados, ambos os métodos estatísticos revelaram que não houve mudança significativa entre as fases de baseline e intervenção.

As informações contidas na Tabela 2 demonstram que houve concordância entre os métodos estatísticos apenas em um dos cinco resultados obtidos por cada método (concordância de 20\%). Diante da discordância entre os métodos estatísticos para a maioria das tarefas investigadas, a análise 
Figura 6

Resultados dos métodos estatísticos Celeration Line e Banda de Dois Desvios-Padrão referente à função manual da criança - sub-teste virar página.

Virar página

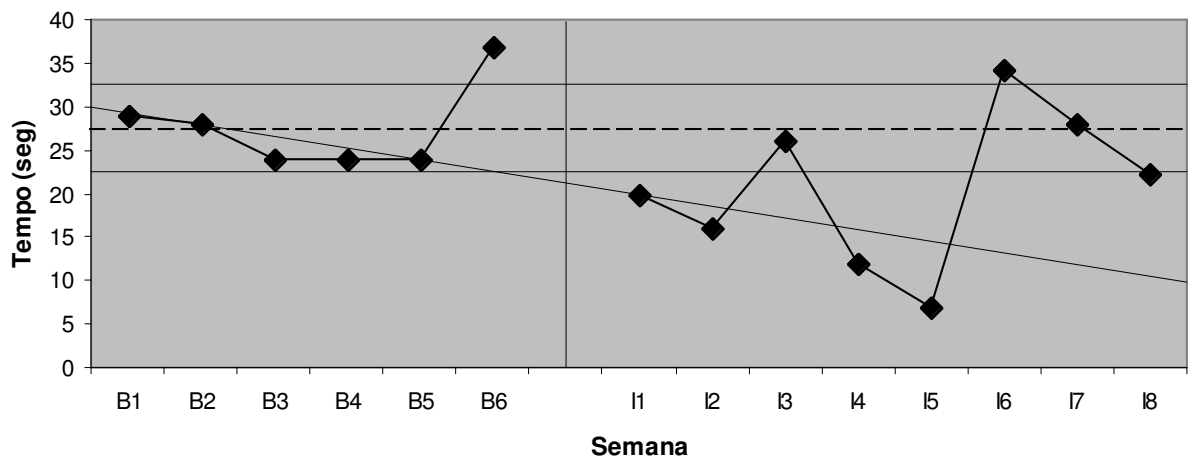

Figura 7

Resultados dos métodos estatísticos Celeration Line e Banda de Dois Desvios-Padrão referente à função manual da criança - sub-teste pegar objetos pequenos.

Pegar Objetos

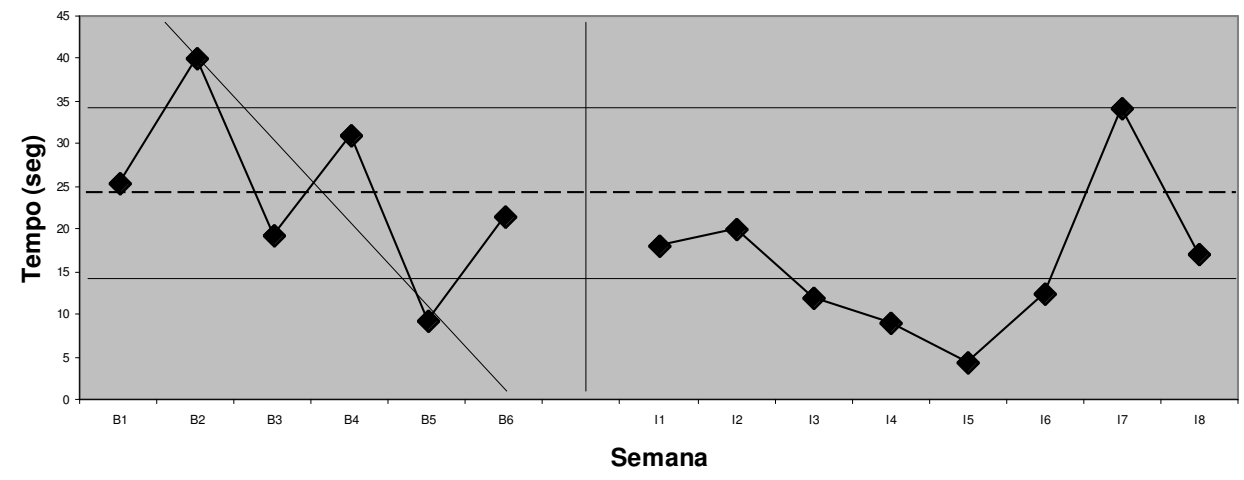

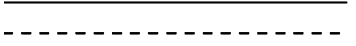

Banda Dois Desvios Padrões

Celeration Line 
Resultados dos métodos estatísticos Celeration Line e Banda de Dois Desvios-Padrão referente à função manual da criança - sub-teste empilhar blocos.

Empilhar blocos

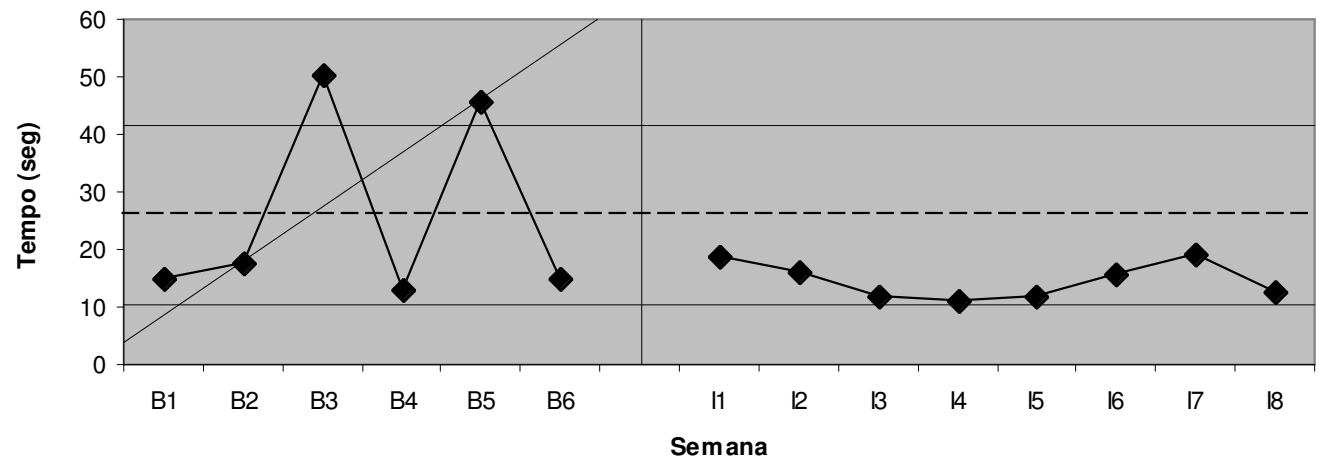

Figura 9

Resultados dos métodos estatísticos Celeration Line e Banda de Dois Desvios-Padrão referente à função manual da criança - sub-teste pegar latas vazias.

Pegar latas vazias

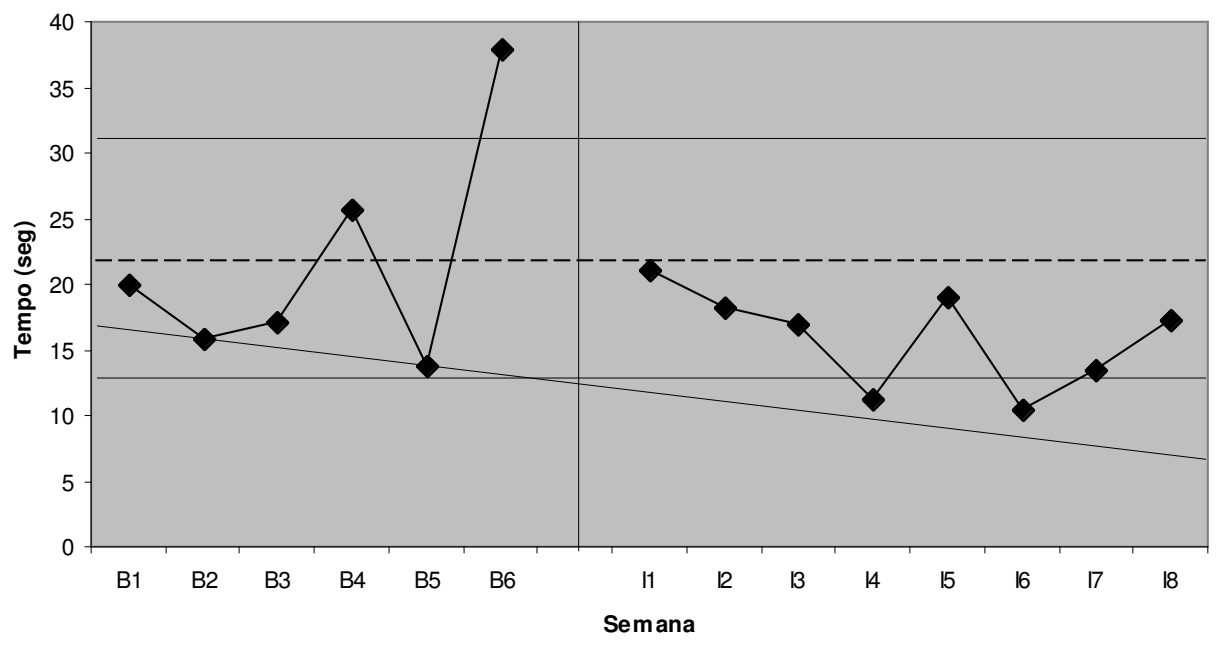

Banda Dois Desvios Padrões

Celeration Line 
Resultados dos métodos estatísticos Celeration Line e Banda de Dois Desvios-Padrão referente à função manual da criança - sub-teste pegar latas cheias.

\section{Pegar latas cheias}
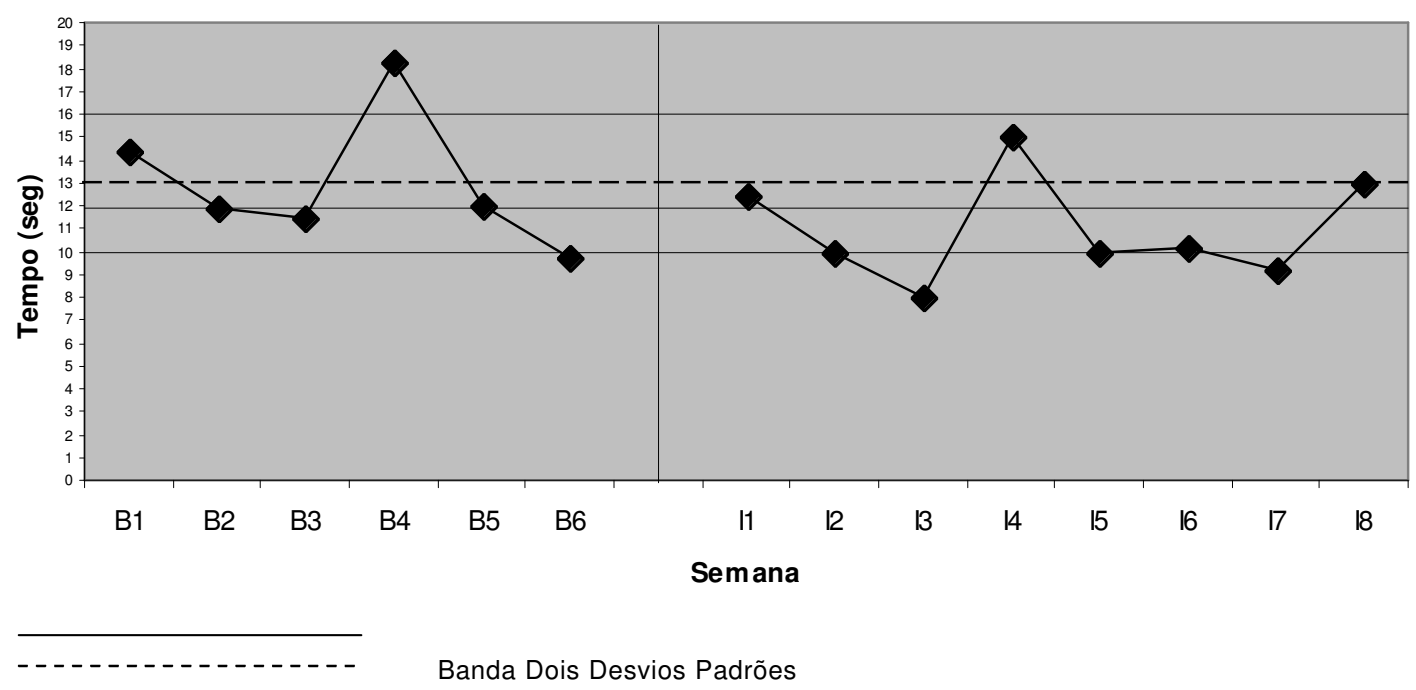

Celeration Line

Tabela 2

Análise dos métodos estatísticos Celeration Line e Banda de Dois Desvios-Padrão das variáveis dependentes função manual da criança.

\begin{tabular}{lccc}
\hline Variáveis dependentes & Celeration Line* & Banda de Dois Desvios-Padrão & Análise visual \\
\hline Virar cartas & NS $(0.363)$ & $\mathrm{S}$ & NS \\
Pegar pequenos objetos & $\mathrm{S} \downarrow(0.004)$ & $\mathrm{S}$ & $\mathrm{NS}$ \\
Empilhar blocos & $\mathrm{S}(0.004)$ & $\mathrm{NS}$ & $\mathrm{S}$ \\
Pegar objetos leves & $\mathrm{S} \downarrow(0.004)$ & $\mathrm{NS}$ & $\mathrm{S}$ \\
Pegar objetos pesados & NS $(0.363)$ & $\mathrm{NS}$ & - \\
\hline
\end{tabular}

$\overline{p<0,05 ;} \mathrm{S}=$ resultado estatisticamente significativo; $\mathrm{\downarrow} \downarrow=$ resultado estatisticamente significativo no sentido oposto ao esperado; NS=resultado estatisticamente não significativo.

visual foi utilizada em adição aos métodos estatísticos para decisão de resultado.

Para a atividade de virar cartas, a análise visual demonstrou uma mudança de nível entre a fase de baseline (tempo médio de 27,60 segundos) e a fase de intervenção (tempo médio de 20,67 segundos); as fases $A$ e $B$ apresentaram a mesma tendência de aceleração sendo que a inclinação da linha de tendência na fase $B$ foi maior. Esperava-se que a linha de tendência tivesse direções opostas e com maior inclinação na fase $B$, o que não ocorreu nesse teste, indicando, portanto, um menor percentual de mudança quando o uso da órtese é iniciado. Nesse caso, a análise visual corrobora os resultados obtidos pelo método Celeration Line (Tabela 2).

Para a tarefa de pegar objetos pequenos, os dois 
métodos estatísticos evidenciaram resultados significativos, porém com direções opostas. Enquanto a Banda de Dois Desvios-Padrão identificou melhora significativa nesta tarefa, a Celeration Line evidenciou declínio significativo. No entanto, a análise visual aponta para uma mudança de nível entre a fase de baseline (tempo médio de 24,21 segundos) e a fase de intervenção (tempo médio de 15,92 segundos) e uma diferença na direção de mudança (desaceleração para aceleração) com menor inclinação da linha de tendência na fase B. Nesse caso, a análise visual corrobora os resultados obtidos pelo método Celeration Line.

Com relação à atividade de empilhar blocos, a análise visual aponta para uma mudança de nível entre a fase de baseline (tempo médio de 26,16 segundos) e a fase de intervenção (tempo médio de 14,17 segundos); a fase de baseline apresentou uma tendência de aceleração e a fase de intervenção apresentou uma tendência à estabilidade (Figura 8). Nesse caso, a análise visual aponta para uma melhora significativa na fase de intervenção, corroborando com os resultados obtidos pelo método Celeration Line (Tabela 2).

Para a atividade de pegar objetos leves, a análise visual demonstrou uma mudança de nível entre a fase de baseline (tempo médio de 21,80 segundos) e a fase de intervenção (tempo médio de 15,99 segundos). As fases $A$ e $B$ apresentaram tendências opostas, sendo a primeira de aceleração e a segunda de desaceleração (Figura 7). Nesse caso, a análise visual aponta para uma melhora significativa na fase de intervenção, não corroborando os resultados obtidos em qualquer dos métodos estatísticos utilizados (Tabela 2).

\section{Discussão}

O presente estudo apresenta informações longitudinais a respeito dos efeitos do uso da órtese de abdução do polegar na amplitude de movimento da mão e função manual de uma criança com paralisia cerebral do tipo hemiparética espástica leve.

Os resultados desse estudo experimental de caso único sugerem que o uso da órtese de abdução de polegar pode levar ao aumento da amplitude de movimento ativa da mão em crianças com paralisia cerebral espástica leve. Na fase de intervenção, a criança aumentou significativamente a amplitude de movimento ativa de extensão e flexão de punho, abdução do polegar e oponência do polegar.

A órtese utilizada na fase de intervenção tem como função principal o posicionamento do polegar de forma a facilitar a abdução e oponência do polegar. Segundo Colditz, ${ }^{3}$ esse fato ocorre porque durante a oposição, a metacarpofalangeana (MCP) do polegar desvia radialmente aproximadamente $20^{\circ}$ e roda levemente para auxiliar no encontro entre a polpa do polegar com o indicador. A mobilidade combinada da MCP com a carpometacarpo (CMC) do polegar permite uma maior amplitude funcional, pois a abdução ocorre simultaneamente em ambas as articulações. ${ }^{3}$

No presente estudo nota-se que esse posicionamento além de influenciar na amplitude de movimento de oponência e abdução de polegar, também influenciou positivamente na movimentação do punho. Tais achados vão ao encontro dos resultados obtidos por Goodman e Basyk ${ }^{10}$ que observaram um aumento significativo dos aspectos funcionais fundamentais da mão (ADM, força de pinça e preensão) após o uso da órtese oponente curta do polegar por quatro semanas, em crianças com paralisia cerebral. Segundo trabalho de Casey e $\mathrm{Kratz}^{7}$ esse tipo de órtese utiliza um dos pontos-chaves propostos pelo método Bobath, o que possibilita um posicionamento funcional, com conseqüente mobilidade dentro de uma determinada amplitude de movimento. Esses autores $^{7}$ sugerem que o uso do ponto-chave como base para posicionamento, possibilita um melhor alinhamento biomecânico do polegar com conseqüente melhora da amplitude de movimento.

Com relação ao desempenho funcional dois aspectos devem ser considerados. Primeiro, o pressuposto de que a melhora da amplitude de movimento deveria influenciar positivamente na funcionalidade da criança e, segundo, que o adequado posicionamento do polegar contribui para um melhor desempenho nas tarefas do cotidiano.

Segundo Erhardt e Merrill ${ }^{24}$ a ADM restrita limita a manipulação e retarda o desenvolvimento das habilidades necessárias para brincadeiras e cuidados pessoais. Considerando que, neste estudo, a criança obteve uma melhora significativa da amplitude de movimento ativa de punho e polegar seria de se esperar que esse resultado influenciasse positivamente na função manual. No entanto, os resultados obtidos apontam para uma melhora da função manual apenas para a tarefa de empilhar blocos. Nas outras tarefas avaliadas, embora tenha sido observada a redução do tempo necessário para sua realização, essa não foi considerada significativa.

Nota-se que a ADM existente na fase de baseline foi suficiente para que a criança executasse, mesmo com dificuldade, as tarefas solicitadas. Embora a melhora da amplitude de movimento ativo tenha sido significativa, este aspecto parece não ter influenciado 
positivamente na melhora do desempenho da criança. Esse dado corrobora o pressuposto de que para a realização da maioria das atividades do cotidiano é necessário que o indivíduo apresente apenas o que se denomina de amplitude de movimento funcional. ${ }^{25-27}$

Com relação à influência do posicionamento do polegar sobre o desempenho funcional da criança, nota-se que esse aspecto variou de acordo com o tipo de preensão necessária para a execução do teste de função manual de Jebsen-Taylor. Os principais tipos de preensão envolvidos no sub-testes são preensão palmar/três pontos (empilhar blocos), polpa-polpa (pegar pequenos objetos e virar cartas) e esférica (pegar objetos leves e pesados).

A preensão palmar (três pontos) exige oposição do polegar em relação aos outros dedos, onde o componente mais importante é a rotação do polegar que permite o contato das polpas digitais. ${ }^{4}$ Neste estudo, o posicionamento do polegar parece ter sido adequado para possibilitar melhora significativa na tarefa de empilhar blocos.

A preensão polpa-polpa exige flexão da interfalangeana do polegar e interfalangeanas proximais e distais dos dedos. Esse tipo de preensão raramente exige a manutenção de postura estática, portanto é um padrão difícil de ser substituído. No entanto, algumas vezes é possível utilizar a preensão palmar para proporcionar maior área de superfície para manter o objeto. ${ }^{4}$ A preensão polpa-polpa necessária para a execução das tarefas de pegar objetos pequenos e virar cartas não foi facilitada pelo posicionamento do polegar. Esses sub-testes exigem maior destreza e manipulação e, no caso específico de virar cartas, associação com o movimento de pro-supinação do antebraço. Tais aspectos podem estar prejudicados pela dificuldade de controle dos movimentos devido à leve espasticidade.

$\mathrm{Na}$ preensão esférica (pegar objetos leves e pesados), os dois dedos ulnares assumem maior flexão permitindo o acoplamento do objeto na palma. Nesse caso, segundo Belkin et al., ${ }^{4}$ mais importante que o posicionamento do polegar é a estabilização de punho para possibilitar a posição da mão em posição intrínsico-plus e atuação efetiva dos músculos lumbricais. Pode-se supor, portanto, que embora o polegar estivesse posicionado adequadamente a criança não apresentava controle suficiente para estabilização do punho necessário ao desempenho dessas tarefas. Além disso, com relação ao sub-teste de pegar objetos pesados, muitas crianças com paralisia cerebral não têm integração funcional sensório-motora durante as tarefas de preensão. A geração de força é geralmente instável com alta margem de segurança e grande variação. Isso faz a criança sentir-se insegura quando carrega objetos em diferentes ambientes, necessitando focar sua atenção à tarefa buscando estratégias compensatórias para sua pobre coordenação de força desenvolvida. ${ }^{7}$ Segundo Casey e Kratz, ${ }^{7}$ somente a partir dos dois anos é que a criança começa a utilizar (escalar) as forças necessárias para diferentes pesos, atingindo os patamares próximos desta função no adulto com a idade de seis a oito anos. Crianças mais novas que três anos não são capazes de controlar o output motor de acordo com o tamanho, mas podem usar um grande percentual de força para objetos pesados. Essa questão também pode ter contribuído para dificultar o desempenho funcional nesta tarefa.

Ainda com relação ao posicionamento do polegar e a melhora funcional, deve-se considerar que embora o uso da órtese possa maximizar a preensão funcional, o grau de abdução e oposição em que o polegar é posicionado pode influenciar os resultados. ${ }^{4}$ Pode-se supor, então, que o posicionamento do polegar diferente do utilizado nesse estudo pode gerar resultados diferentes dos obtidos aqui.

As observações durante a coleta de dados e a melhora, embora não significativa, no tempo gasto para completar as tarefas sugerem melhoras qualitativas no desempenho motor. $\mathrm{O}$ teste de função manual de Jebsen-Taylor foi padronizado para avaliar a função manual de crianças com paralisia cerebral, no entanto, ao utilizar apenas o tempo como parâmetro de medida, não possibilita inferir sobre mudanças relacionadas a outros aspectos da função manual. $\mathrm{Na}$ literatura observa-se uma escassez de testes padronizados para avaliar a função manual de crianças com paralisia cerebral e, dentre os instrumentos disponíveis, verificou-se que o teste de Jebsen-Taylor era o mais específico e viável para mensurar a função manual por basear-se em medidas objetivas de tarefas funcionais que freqüentemente são utilizadas em atividades de vida diária dessas crianças.

Outra questão a ser levantada é a consistência dos resultados entre os métodos estatísticos utilizados. Os achados desse estudo demonstram que a consistência dos resultados entre os métodos estatísticos Celeration Line e Banda de Dois Desvios-Padrão, utilizados para avaliar o uso da órtese e a função manual foi limitada. Os dois procedimentos obtiveram resultados consistentes em apenas $37,5 \%$ dos resultados. Nourbakhsh e Ottenbacher ${ }^{23}$ ao compararem três diferentes métodos de análises estatísticas (Celeration Line, Banda de Dois Desvios-Padrão, Estatística C) utilizados em estudos de caso-único, evidenciaram um reduzido índice de concordância entre eles (38\%). Entre os métodos Celeration Line e Banda de Dois DesviosPadrão, o índice de concordância foi de apenas $48 \%$. 
A concordância obtida nesta investigação apresentouse abaixo daquela obtida por aqueles autores.

A utilização de métodos quantitativos em investigações com desenhos de caso único ainda é uma prática relativamente recente. Embora alguns pesquisadores defendem o uso desses procedimentos em substituição à análise visual, ${ }^{28}$ questões a respeito de qual o melhor método a ser aplicado, da equivalência entre diferentes procedimentos estatísticos e das vantagens e limitações de cada método permanecem sem consenso. Em virtude disso, foram considerados somente os resultados corroborados pela análise visual, como recomendado por Nourbakhsh e Ottenbacher. ${ }^{23}$

Embora o estudo de caso único possibilite examinar cuidadosamente os efeitos do uso da órtese e com considerável detalhamento, seu desenho limita a generalização dos resultados para uma população. Um estudo randomizado envolvendo uma amostra maior e mais diversa é necessário para determinar o efeito deste dispositivo no desempenho funcional. O uso de um grupo controle é fundamental para determinar que mudanças na função podem ser atribuídas à intervenção e quais são secundárias ao processo contínuo do desenvolvimento motor e de outras intervenções terapêuticas. Apesar da limitação, de um estudo AB, esse tipo de desenho metodológico é o único que informa sobre o padrão de mudanças observado em um indivíduo após a administração de uma intervenção. Tal informação é relevante para definição de tempo necessário para evidenciar mudanças, bem como do perfil de mudanças resultante dos efeitos da terapêutica de interesse.

\section{Conclusões}

O uso da órtese de abdução do polegar teve um efeito significativo na melhora da amplitude de movimento da mão na criança com paralisia cerebral espástica hemiparética leve. Com relação ao uso da órtese no desempenho funcional da criança em tarefas específicas, o estudo realizado não pode inferir que esse dispositivo tem efeito positivo no desempenho das tarefas investigadas dado o pequeno grau de concordância entre os métodos de análise estatísticos realizados. Essas informações podem ser úteis aos profissionais que trabalham com essa clientela, auxiliando-os no processo de intervenção. Embora os resultados desse estudo sejam encorajadores, pesquisas futuras com desenhos controlados e randomizados são necessárias para determinar a eficácia do uso desse dispositivo com uma grande população.

\section{Referências}

1. Pehoski C. Object manipulation in infants and children. In: Henderson A, Pehoski C, editors. Hand function in the child: foundations for remediation. St Louis: Mosby; 1995. p. 136-53.

2. Case-Smith J. Grasp, release, and bimanual skills in the first two years of life. In: Henderson A, Pehoski C, editors. Hand function in the child: foundations for remediation. St Louis: Mosby; 1995. p. 113-35.

3. Colditz JC. Anatomic considerations for splinting the thumb. In: Hunter JM, Mackin EJ, Callahan AL, Osterman AL, Skirven TM, Schineider LH, Rich RR, editors. Rehabilitation of the hand and upper extremity. St. Louis: Mosby; 2005. p. 1858-75.

4. Belkin J, English CB, Adler C, Pedretti LW. Orthotics. In: Pedretti LW, editor. Occupational therapy - Practice skills for physical dysfunction. St. Louis: Mosby; 1996. p. 31950.

5. Fess EE, Philips CA. Hand splinting: principles and methods. St. Louis: Mosby; 1987.

6. Crocker MD, Mackay-Lyons M, McDonnell E. Forced use of the upper extremity in cerebral palsy: a single-case design. Am J Occup Ther. 1997; 51: 824-33.
7. Casey CA, Kratz EJ. Soft splinting with neoprene: the thumb abduction supinator splint. Am J Occup Ther. 1988; 42: $395-8$.

8. Kinghorn J, Roberts G. The effect of inhibitive weightbearing splint on tone and function: a single-case study. Am J Occup Ther. 1996; 50: 807-15.

9. Eliasson AC. Sensorimotor integration of normal and impaired development of precision movement of the hand. In: Henderson A; Pehoski C, editors. Hand function in the child: foundations for remediation. St Louis: Mosby; 1995. p. $40-54$.

10. Goodman G, Bazyk S. The effects of a short thumb opponents splint on hand function in cerebral palsy: a singlesubject study. Am J Occup Ther. 1991; 45: 726-31.

11. Wilton J. Casting, splinting, and physical and occupational therapy of hand deformity and dysfunction in cerebral palsy. Hand Clin. 2003; 19: 573-84.

12. Hogan L, Uditsky T. Pediatric splinting - selection, fabrication and clinical application of upper extremity splints. Texas: Therapy Skill Builders; 1998.

13. Aguiar IF, Rodrigues AMVN. O uso de órteses no tratamento de crianças com paralisia cerebral na forma hemiplégica. Temas Desenvolv. 2002; 11: 45-51. 
14. Ashworth B. Preliminary trial of carisoproal in multiple sclerosis. Practioner. 1964; 192: 540-2.

15. American Society of Hand Therapists. Clinical assessment recommendations. Chicago; 1992.

16. Cambridge-Keeling CA. Range-of-motion measurement of hand. In: Mackin EJ, Callahan AD, Skirven TM, Schineider LH, editors. Rehabilitations of the hand and upper extremity. St Louis: Mosby; 2002. p. 169-82.

17. Stern E. Stability of Jebsen Taylor hand function test across three test sessions. Am J Occup Ther. 1992; 46: 647-9.

18. Hackel M, Wolfe G, Bang S, Canfield J. Changes in hand function in the aging adult as determined by Jebsen test of hand function. Phys Ther. 1992; 72: 373-7.

19. Jebsen R, Taylor N, Trieschmann RB, Trotter MJ, Howard LH. An objective and standardized test of hand function. Arch Phys Med Rehabil. 1969; 6: 311-9.

20. Taylor N, Sand PL, Jebsen RH. Evaluation of hand function in children. Arch Phys Med Rehabil. 1973; 54: 129-35.

21. Carlson J, Trombly C. The effect of wrist immobilization on performance of the Jebsen hand function test. Am J Occup Ther. 1983; 33: 167-75.
22. Portney LG, Watkins MP. Foundations of clinical research applications to practice. New Jersey: Prentice Hall Health Upper Saddle River; 2000.

23. Nourbakhsh MR, Ottenbacher KJ. The statistical analysis of single-subject data: a comparative examination. Phys Ther. 1994; 74: 768-76.

24. Erhardt RP, Merrill SC. Disfunção neurológica em crianças. In: Neistadt ME, Crepeau EB, editores. Terapia ocupacional. Rio de Janeiro: Guanabara Koogan; 2002. p. $542-$ 66.

25. Kohlmeyer K. Avaliação dos componentes de desempenho. In: Neistadt ME, Crepeau EB, editores. Terapia ocupacional. Rio de Janeiro: Guanabara Koogan; 2002. p. 20365.

26. Trombly CA. Occupational therapy for physical disfunction. Baltimore: Williams \& Wilkins; 1995.

27. Pedretti LW, Early MB. Terapia ocupacional - capacidades práticas para as disfunções físicas. São Paulo: Roca; 2004.

28. Jones RR, Weinrott MR, Vaught RS. Effects of serial dependency on agreement between visual and statistical inferences. J Appl Behav Anal. 1978; 11: 277-83.

Recebido em 8 de novembro de 2006

Versão final apresentada em 2 de agosto de 2007

Aprovado em 26 de agosto de 2007 